\title{
Analysis of Rough Gear Gashing Force for Multi-engaged Cutting Blades
}

\author{
ZHANG Jin $^{1, a^{*}}$, ZHANG Jianrun ${ }^{1, b}$,SUN Yuejiang ${ }^{2, c}$, HUANG Xiaodiao ${ }^{3, d}$ and \\ DAI Yongfen ${ }^{2, e}$ \\ ${ }^{1}$ Southeast University, No. 2 Sipailou, Nanjing, 210096, China \\ ${ }^{2}$ Ma'anshan Fangyuan slewing ring Co., Ltd, Chaoshan Road, Ma'anshan, 243052, China \\ ${ }^{3}$ Nanjing Tech. University, No. 5 Xinmofan Road, Nanjing, 210009, China \\ azhangjin2002888@163.com, ${ }^{b}$ zhangjr@seu.edu.cn, ${ }^{c}$ syj@masfy.com, ${ }^{d}$ njgdhxd@189.cn, \\ gy@masfy.com
}

\begin{abstract}
Keywords: Disk-type gear gasher; Chip segment; Profile time.
Abstract. Disk-type gear gasher is promised by a cutter body and indexable inserts, affording gear gashing which belongs to no free, heavy cutting. By applying nonlinear empirical exponential formula and using Time Finite Element Analysis method, the model of gashing forces was obtained. The corresponding gashing torque was unanimity as the spindle current measured. Based on the intermittent force model, the machining condition was focused along the gear lead for the forces' amplitude and phase on the profile time. The value changed greatly until 0 . There were two manifestations called phase congruency and phase imbalance which led to negative deviations or positive deviations. So, the force of rough gear gashing had been completed preliminarily, providing guidance for processing.
\end{abstract}

\section{Introduction}

Disk-type gear gasher can process big modulus gear efficiently. It has become popularity processing method over the past decade that can remove larger amount of material than conventional methods such as hobbing, shapping and leads to a new market, resulting industrial innovation. The mechanism of metal cutting has been of interest to a lot of researchers [1-4]. Models of the cutting forces, surface location error, vibration, tool wear, and regeneration for the intermittent cutting process were proposed [5,6]. The gear gashing as a unique milling process has the characteristics of non-free, multi-blades, intermittent cutting, air-cooled and variable chip thickness. Since the profile of the blade forms the gear contour, it isn't the same as end milling, ball milling, face milling, etc. The diameter is generally about $350 \mathrm{~mm}$ to $480 \mathrm{~mm}$ which is larger than that of the conventional ones and equivalent to the workpiece. The corresponding rote speed is approximately $100 \mathrm{rpm}$ with significant differences from the end mill, which can reach at least up to $1000 \mathrm{rpm}$. Time Finite Element Analysis (TFEA) was used as a sound reasonable method for interrupted cutting [7,8]. Andersson presented a mechanical cutting force model for band-sawing which described the forces between individual teeth and related it to initial positional errors, tool dynamics and edge wear [9]. Zatarain considered the influence of the helix angle and the consequent phase lag between the forces appearing at different sections [10]. Domnita evaluated the near-dry machining effects on the gear hobbing with respect to hob wear, surface quality, cooling effect, and environment protection [11]. Zhang proposed an index empirical model to calculate the cutting torque by integrating the torque of segmental edges [12]. Above work provides great help. However, further reach should be given to more accurate model, machining accuracy, and so on.

\section{Force calculation}

\section{Model of disk-type gear gasher}

Rough gear gashing system contains a headstock, a gear gasher, a workpiece and a fixture. A disk-type gear gasher generally includes the cutter body and indexable inserts with two or four blades. Mostly, the axial cross-section is promised by simple lines and arcs. It can process a type of modulus 
with different teeth quality. Generally speaking, an acceptable gasher should remove most of the material on the precondition of high efficiency, even allowance, and great precision. In the derivation of the gashed gear slot, the relative motion of the coordinate system is shown in Fig. $1 . O-X Y Z$ is fastened to the machine, $O_{\mathrm{c}}-X_{\mathrm{c}} Y_{\mathrm{c}} Z_{\mathrm{c}}$ to the cutter, $O_{\mathrm{w}}-X_{\mathrm{w}} Y_{\mathrm{w}} Z_{\mathrm{w}}$ to the workpiece, and $O_{\mathrm{s}}-X_{\mathrm{s}} Y_{\mathrm{s}} Z_{\mathrm{s}}$ to the cross-section of the slot. $A K E$ is the section of $R_{1}$ blade that includes a top edge $A K$, a side edge $K E$ and a transition point $K$. Only the side edge works in $R_{2}$ and $R_{3}$. Points $E$ and $F$ are transitions. The combination of $R_{1}, R_{2}$ and $R_{3}$ approaches the gear contour. Similarly, the left side blades are noted $L_{1}$ $L_{2} L_{3}$, and so on. Process allowance between the machined contour and the theoretical gear contour can be seen, which is suitable for a subsequent finishing process. The gashing depth is $h$ and the gear height is $H$. The cross-section of the cutter is shown in Fig. 1c. There are two main motions in spur gear gashing. One motion is $n$, and the other is $v . \varphi$ is rotation angle of the blade in $O_{\mathrm{s}}-X_{\mathrm{s}} Y_{\mathrm{s}}$. Feed per tooth is $f_{\mathrm{t}}$. The diameter of the gasher is $R_{0}$. The chip topology based on the processing completed previously was shown in Ref. [12].

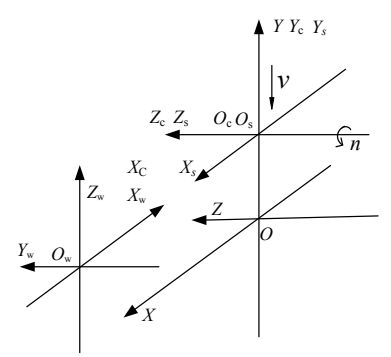

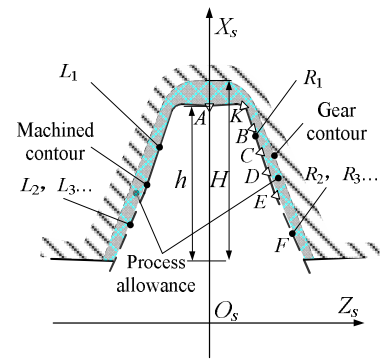

(b)

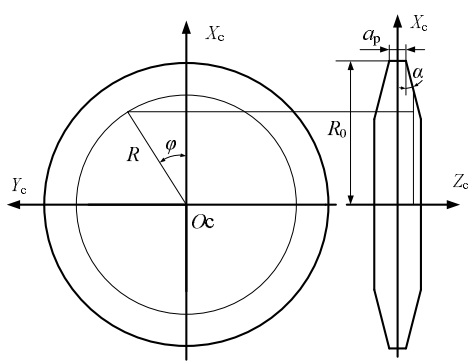

(c)

Fig. 1 (a) Gashing System coordinates (b) Cross-section of the slot (c) Basic cross-section of disk-type gear gasher

Different effective blades for each kind of insert distribute evenly in a circle. A convenient definition of an evenly arranged blade is one that names 'left - right ... - left - right'. Examples include ' $L_{11}-R_{21}-L_{21}-R_{11}$ ', ' $L_{11}-L_{12}-R_{31}-R_{21}-L_{31}-R_{11}-L_{21}-R_{12}$ ', and so on. The first subscripted number represents the blade type and the second one is the serial number. A kind of rough gasher is shown in Table 1.

Table 1 Inserts layout

\begin{tabular}{cccccc}
\hline Item & Location $\theta /\left(^{\circ}\right)$ & Effective cutting depth $/ \mathrm{mm}$ & Item & Location $\theta /\left(^{\circ}\right)$ & Effective cutting depth $/ \mathrm{mm}$ \\
\hline$L_{11}$ & 0 & 25 & $R_{21}$ & 0 & 15 \\
$L_{21}$ & 18 & 15 & $R_{11}$ & 18 & 25 \\
$L_{12}$ & 36 & 25 & $R_{22}$ & 36 & 15 \\
$L_{22}$ & 54 & 15 & $R_{12}$ & 54 & 25 \\
$\ldots$ & $\ldots$ & $\ldots$ & $\ldots$ & $\ldots$ & $\ldots$ \\
$L_{110}$ & 324 & 25 & $R_{210}$ & 324 & 15 \\
$L_{210}$ & 342 & 15 & $R_{110}$ & 342 & 25 \\
\hline
\end{tabular}

\section{Calculation model of gashing force}

A model which was capable of calculating the gashing force was proposed by using nonlinear empirical exponential formula with TFEA method. Discrete the cutting edge into micro-segments, calculate the corresponding micro-segment chip layer at any time, obtain the thickness and length of the chip, and acquire the material remove rate. Then, the gashing force as nonlinear exponential equation for each segment can be obtained every segmented time scale. The forces for the minimum cycle of a typical blade can be synthesised by the integral method. Next, generate all along the whole cause on the phase change and vector superposition principle. Since top edge and side one of the gasher are different, the calculation models are presented respectively.

(1) Because top edge $A K$ is parallel to the axis and the length is $a_{\mathrm{p}}$, all segments can be projected to a point as shown in Fig. 2. The tangential force $\mathbf{F}_{\mathrm{Tt}}$ is opposite to the speed direction and calculated as follows: 


$$
\mathbf{F}_{\mathrm{Tt}}=\left\{\begin{array}{l}
F_{\mathrm{Ttx}} \\
F_{\mathrm{Tty}} \\
F_{\mathrm{Ttz}}
\end{array}\right\}=\left\{\begin{array}{c}
u \cdot f_{t} \cdot \sin ^{2} \varphi \cdot a_{\mathrm{p}} \\
u \cdot f_{t} \cdot \sin \varphi \cdot \cos \varphi \cdot a_{\mathrm{p}} \\
0
\end{array}\right\}
$$

The radial force $\mathbf{F}_{\mathrm{Tr}}$ is perpendicular to $\mathbf{F}_{\mathrm{Tt}}$ and $A K$ and its value has a linear correlation $C$ with $F_{\mathrm{Tt}}$.

$$
\mathbf{F}_{\mathrm{Tr}}=\left\{\begin{array}{l}
F_{\mathrm{Trx}} \\
F_{\mathrm{Try}} \\
F_{\mathrm{Trz}}
\end{array}\right\}=C \cdot\left\{\begin{array}{c}
-u \cdot f_{t} \cdot \sin \varphi \cdot \cos \varphi \cdot a_{\mathrm{p}} \\
u \cdot f_{t} \cdot \sin ^{2} \varphi \cdot a_{\mathrm{p}} \\
0
\end{array}\right\}
$$

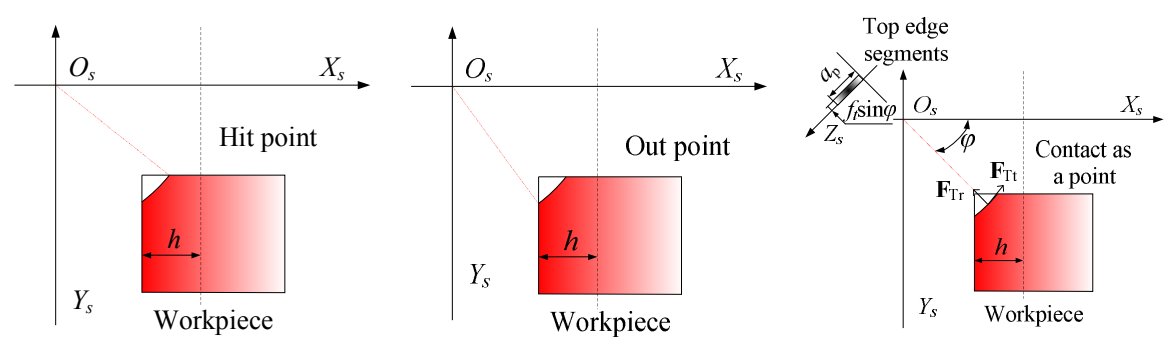

(a)

(b)

(c)

Fig. 2 Segment of top edge (a) Hit point (b) Out point (c) Contact as a point

(2) Side edge $K E$ is projected as a line as shown in Fig. 3. Cutting segment changed over time. The tangential force $\mathbf{F}_{\mathrm{St}}$ is calculated as follows.

$$
\mathbf{F}_{\mathrm{St}}=\left\{\begin{array}{l}
F_{\mathrm{Stx}} \\
F_{\mathrm{Sty}} \\
F_{\mathrm{Stz}}
\end{array}\right\}=\left\{\begin{array}{c}
\int_{z_{0}}^{z_{1}} u \cdot\left(f_{t} \cdot \sin \varphi \cdot \frac{\mathrm{d} f(R)}{\mathrm{d} R}\right) \cdot \sin \varphi \cdot \sqrt{1+\left(\frac{\mathrm{d} f(R)}{\mathrm{d} R}\right)^{2}} \mathrm{~d} Z_{\mathrm{s}} \\
\int_{z_{0}}^{z_{1}} u \cdot\left(f_{t} \cdot \sin \varphi \cdot \frac{\mathrm{d} f(R)}{\mathrm{d} R}\right) \cdot \cos \varphi \cdot \sqrt{1+\left(\frac{\mathrm{d} f(R)}{\mathrm{d} R}\right)^{2}} \mathrm{~d} Z_{\mathrm{s}} \\
0
\end{array}\right\}
$$

Where $u$ is force per unit volume under a specific temperature and chip thickness. $z_{0}$ and $z_{1}$ are contact locations between side edge $K E$ and workpiece and can be got at every moment.

$$
u=1000 \times e^{b} \cdot\left(f_{t} \cdot \sin \varphi \cdot \frac{\mathrm{d} f(R)}{\mathrm{d} R}\right)^{a}
$$

Where $a$ and $b$ are the gashing force constant for $u$.

Generally, $K E$ profile in Fig. 1c is

$$
\left\{\begin{array}{c}
X_{s}=R \sin \varphi \\
Z_{s}=f(R)=\mp\left(\frac{a_{\mathrm{p}}}{2}+\left(R_{0}-R\right) \tan \alpha\right)
\end{array}\right.
$$

Where '-' indicates the tool on the left side and '+' indicates the right side of the tool. $\alpha$ is pressure angle.

The radial force $\mathbf{F}_{\mathrm{Sr}}$ is perpendicular to $\mathbf{F}_{\mathrm{St}}$ and $K E$ and its value has a linear correlation $C$ with $F_{\mathrm{St}}$ as follows:

$$
\frac{\mathbf{F}_{\mathrm{Sr}}}{\left|\mathbf{F}_{\mathrm{Sr}}\right|}=\frac{\mathbf{F}_{\mathrm{St}} \times \overrightarrow{K E}}{|| \mathbf{F}_{\mathrm{St}}|\times| \overline{K E} \mid}
$$




$$
\begin{aligned}
& \mathbf{F}_{\mathrm{Sr}}=\left\{\begin{array}{l}
F_{\mathrm{Srx}} \\
F_{\mathrm{Sry}} \\
F_{\mathrm{Srz}}
\end{array}\right\}=C \cdot \frac{\mathbf{F}_{\mathrm{St}} \times \overrightarrow{K E}}{|\overrightarrow{K E}|}=C \cdot\left\{\begin{array}{l}
-\int_{z_{0}}^{z_{1}} u \cdot\left(f_{t} \cdot \sin \varphi \cdot \frac{\mathrm{d} f(R)}{\mathrm{d} R}\right) \cdot \cos \varphi \frac{\mathrm{d} f(R)}{\mathrm{d} R} \cdot \sqrt{1+\left(\frac{\mathrm{d} f(R)}{\mathrm{d} R}\right)^{2} \mathrm{~d} Z_{\mathrm{s}}} \\
\int_{z_{0}}^{z_{1}} u \cdot\left(f_{t} \cdot \sin \varphi \cdot \frac{\mathrm{d} f(R)}{\mathrm{d} R}\right) \cdot \sin \varphi \frac{\mathrm{d} f(R)}{\mathrm{d} R} \cdot \sqrt{1+\left(\frac{\mathrm{d} f(R)}{\mathrm{d} R}\right)^{2} \mathrm{~d} Z_{\mathrm{s}}} \\
-\int_{z_{0}}^{z_{1}} u \cdot\left(f_{t} \cdot \sin \varphi \cdot \frac{\mathrm{d} f(R)}{\mathrm{d} R}\right) \cdot \frac{\mathrm{d} f(R)}{\mathrm{d} R} \cdot \sqrt{1+\left(\frac{\mathrm{d} f(R)}{\mathrm{d} R}\right)^{2} \mathrm{~d} Z_{\mathrm{s}}}
\end{array}\right\}
\end{aligned}
$$

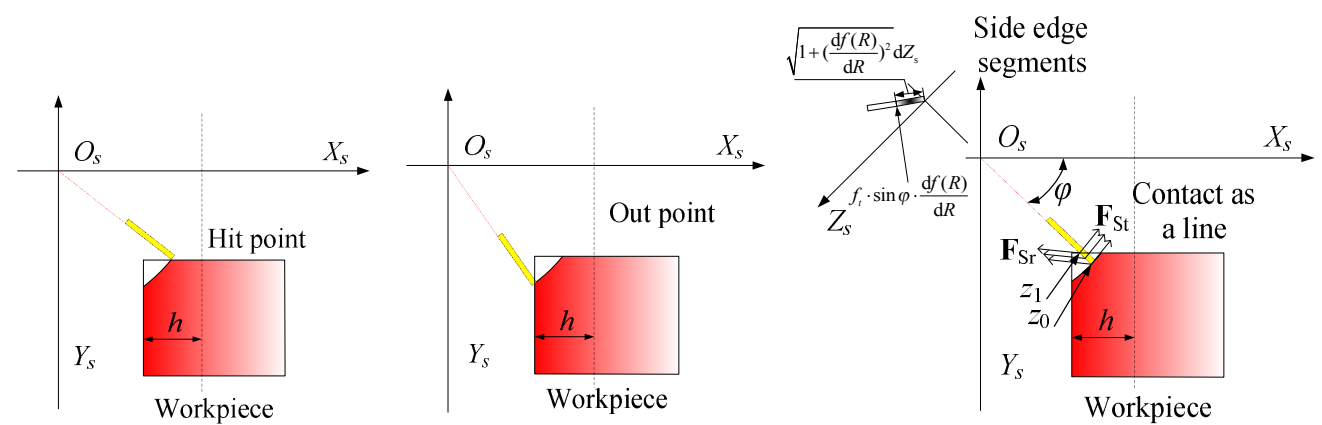

(a)

(b)

(c)

Fig. 3 Segment of side edge (a) Hit point (b) Out point (c) Contact as a line

$\mathbf{F}_{\mathrm{R}}$ of $R_{1}$ can be obtained. Furthermore, $\mathbf{F}_{\mathrm{L}}$ of $L_{1}$ can be acquired by the same method. To facilitate the analysis, the obtained forces are decomposed into the Cartesian coordinate system.

$$
\begin{aligned}
& \mathbf{F}_{\mathrm{R}}=\left\{\begin{array}{l}
F_{\mathrm{Rx}} \\
F_{\mathrm{Ry}} \\
F_{\mathrm{Rz}}
\end{array}\right\}=\left\{\begin{array}{l}
F_{\mathrm{Ttx}}+F_{\mathrm{Trx}}+F_{\mathrm{Stx}}+F_{\mathrm{Srx}} \\
F_{\mathrm{Tty}}+F_{\mathrm{Try}}+F_{\mathrm{Sty}}+F_{\mathrm{Sry}} \\
F_{\mathrm{Ttz}}+F_{\mathrm{Try}}+F_{\mathrm{Stz}}+F_{\mathrm{Sry}}
\end{array}\right\} \\
& \mathbf{F}=\mathbf{F}_{\mathrm{R}}+\mathbf{F}_{\mathrm{L}}=\left\{\begin{array}{l}
F_{x} \\
F_{y} \\
F_{z}
\end{array}\right\}
\end{aligned}
$$

The gasher diameter is generally about $350 \mathrm{~mm}$ to $480 \mathrm{~mm}$, which is larger than the conventional one's and equivalent to the workpiece. For convenience, the entire process can be divided into three stages called the hit, middle and out stage, as shown in Fig. 4. The hit stage is the procedure that performs before the tool's axis reaching the top surface of the gear. The middle stage begins after the hit stage to the out stage when the tool reaches the opposite one of the gear. While, there are three kinds of conditions including vacancy, only one edge and multi-edges engaging with the workpiece. So contact time should be paid attention to. If there is no contact,

$$
F_{i j}=0 \quad i=R, L ; j=x, y, z
$$

So, by using nonlinear empirical exponential formula with TFEA method, the gashing forces can be calculated associated with the inserts layout. Then gashing torque and gashing power can be evaluated. If the blades' locations at some point are the same, axial force $F_{z}$ approaches 0 together for the offset force. But, because of the stagger teeth, $F_{z}$ generated by each blade accumulates Non-zero.

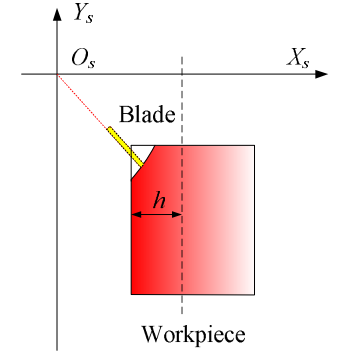

(a)

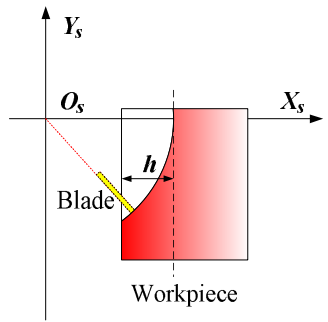

(b)

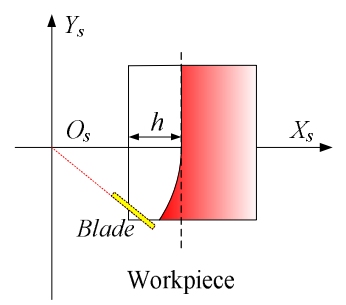

(c)

Fig. 4 Different stages (a) Hit stage (b) Middle stage (c) Out stage 


\section{Analysis of experiment}

\section{Spindle current}

In the cutting force model, the following three coefficients are to be given for a kind of workpiece whose material is $42 \mathrm{CrMo} . a=-0.452, b=0.230, C=0.3$. Table 2 shows other parameters. In the experiment $n=95 \mathrm{rpm}, v=240 \mathrm{~mm} / \mathrm{min}, h=40 \mathrm{~mm}$.

Table 2 Workpiece parameters

\begin{tabular}{cc}
\hline Item & Workpiece \\
\hline Module $/ \mathrm{mm}$ & 20 \\
Teeth number & 138 \\
Pressure angle $/\left(^{\circ}\right)$ & 20 \\
Gear width $/ \mathrm{mm}$ & 136 \\
\hline
\end{tabular}

Spindle current detected from YDF-I alternating current transducer is shown in Fig. 5a. In the macro aspect, the gashing torque is unanimity as the spindle current measured in Fig. 5b. The max. current value is $46.9 \mathrm{~A}$ and the max. output power approximates as $30.9 \mathrm{~kW}$. While the max. gashing torque is $2352 \mathrm{~N} \cdot \mathrm{m}$ and the max. gashing power is about $23.4 \mathrm{~kW}$. So the power factor is 0.76 and realistic. In fact, $F_{z}$ has not consumed energy, unlike $F_{x}$ or $F_{y}$. The gashing forces in three stages are distinguished by Line 1 and Line 2 in Fig. 5c. As the figure illustrates, $F_{x}$ and $F_{y}$ appear in trapezium form, but $F_{z}$ in the interval presents the positive and negative variations. $F_{y}$ is larger than $F_{x}$ because the feed direction is parallel to $Y$ axis.

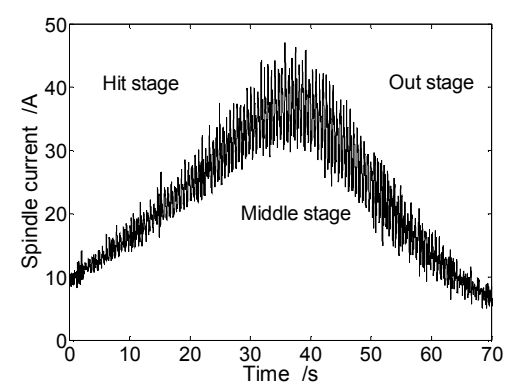

(a)

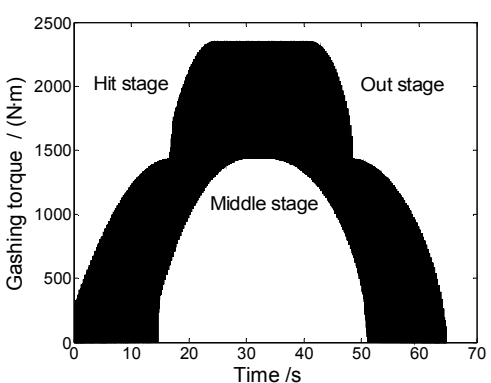

(b)

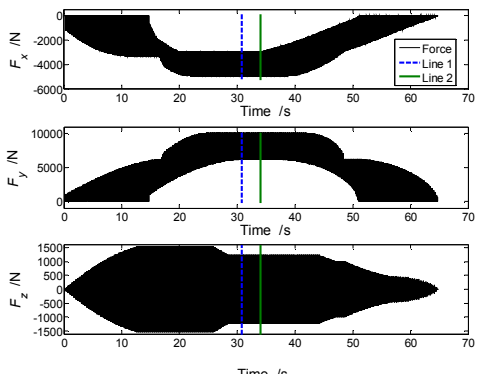

(c)

Fig. 5 (a) Spindle current (b) Gashing torque (c) Gashing forces

\section{Forces on the profile time}

Gashing characteristics such as multi-blades engagement, intermittent cutting and variable chip thickness will influence the machining accuracy. On the transverse direction, gear profile is formed and the machine rigidity is the weakest. To improve the static precision, the machine tool and the gasher should be at high precision level. But the forces affect the dynamic behaviour directly which affect performance greatly. Generally speaking, $F_{x}$ forces the cutter out of the workpiece in the radial direction, which results in a positive deviation and influences the gear profile accuracy and the lead accuracy over time. $F_{z}$ forces the cutter and the workpiece shaking in the tangential direction. Thus, the deviation may be positive or negative. $F_{y}$ affects the operation precision by influencing the other two components. Based on the intermittent force model, the machining condition can be predicted along the gear lead according to the forces' amplitude and phase on the profile time. There are two manifestations called phase congruency and phase imbalance. As shown in Fig. 6, from $30.0 \mathrm{~s}$ to 30.1 $\mathrm{s}$, the largest amplitude and phase congruency appears, resulting negative deviation. From $48.0 \mathrm{~s}$ to $48.1 \mathrm{~s}$, absolute value decreases and the phase maintains consistently. From $55.0 \mathrm{~s}$ to $55.1 \mathrm{~s}$, on the profile moments of both surfaces, all forces decreases to 0, causing little influence. From $60.0 \mathrm{~s}$ to $60.1 \mathrm{~s}$, the load decreases further. Therefore, the lead accuracy trends can be reflected in the four typical charts. If the inserts layout changes, the whole cause will be in another form. 

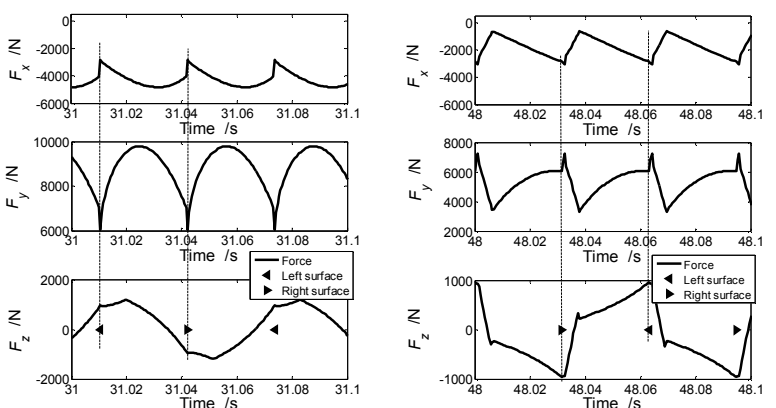

(a)

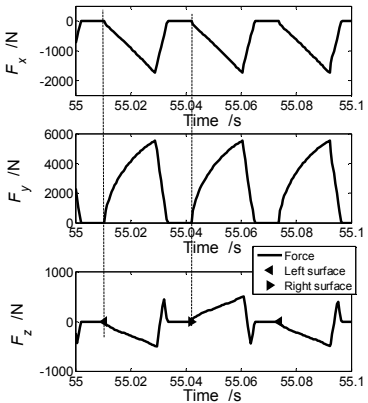

(c)

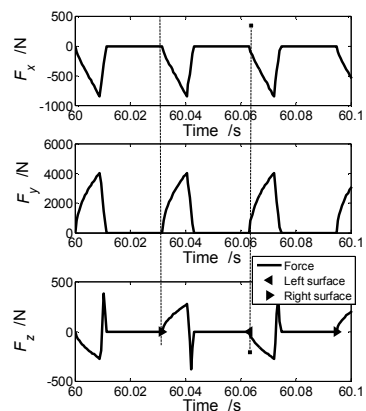

(d)

Fig. 6 Forces on different profile moments (a) $31.0 \sim 31.1 \mathrm{~s} \mathrm{(b)} 48.0 \sim 48.1 \mathrm{~s}$ (c) $55.0 \sim 55.1 \mathrm{~s}$ (d) $60.0 \sim 60.1 \mathrm{~s}$

\section{Conclusions}

Considering the entire gashing process which can be divided into three stages as hit, middle and out stage, a force model of calculation was proposed by using nonlinear empirical exponential formula with TFEA method. Contact time should be paid attention to. There is no force value on the condition of no contact. $\mathrm{F}_{x}$ and $\mathrm{F}_{y}$ appear in trapezium form, and gashing torque was unanimity as the measured spindle current. $F_{\mathrm{z}}$ in the interval presented positive and negative variations. Based on the intermittent force model, the machining condition can be predicted along the gear lead according to the forces' amplitude and phase on the profile time. A comprehensive machining dynamics model of gashing deserves further consideration for machining accuracy.

\section{Acknowledgment}

The authors would like to give thanks to National Natural Science Foundation of China (Grant No. 51175242 ) and Special grand national science-technology project (Grant No. 2012ZX04002-041)

\section{References}

[1] H. Opitz, F. Bernardi: CIRP Annals, Vol. 18(1970), p. 335

[2] J. Tlusty: J. of Eng. for Ind. Vol. 108(1986), p. 59

[3] F. Taylor: Transactions of ASME, Vol. 43 (1907), p. 31

[4] J. Tlusty, M. Polacek: Int. Res. Prod. Eng. Vol. 1 (1963), p. 465

[5] O. A. Bobrenkov, F. A. Khasawneh, and E. A. Butcher: J. Sound Vib. Vol. 329 (2010), p. 585

[6] B. P. Mann, B. T. Edes, and S. J. Easley: Int. J. Mach. Tool Manu. Vol. 48 (2008), p. 350

[7] P. V. Bayly, J. E. Halley, and B. P. Mann: JMSE, Vol. 125 (2003), p. 220

[8] Y. Altintas, G. Stepan, and D. Merdol: CIRP-JMST, Vol. 1 (2008), p. 35

[9] C. Andersson, M. T. Andersson, J. E. Stahl: Int. J. Mach. Tool Manu, Vol. 41 (2001), p. 227

[10] M. Zatarain, J. Muñoa, G. Peigné: CIRP Annals, Vol. 55 (2006), p. 365

[11] F. Domnita: J. Clea. Prod. Vol.17 (2009), p. 839

[12] J. Zhang, X. d. Huang, and Q. Peng: J. Mech. Eng. Vol. 47 (2011), p. 186 (in Chinese) 